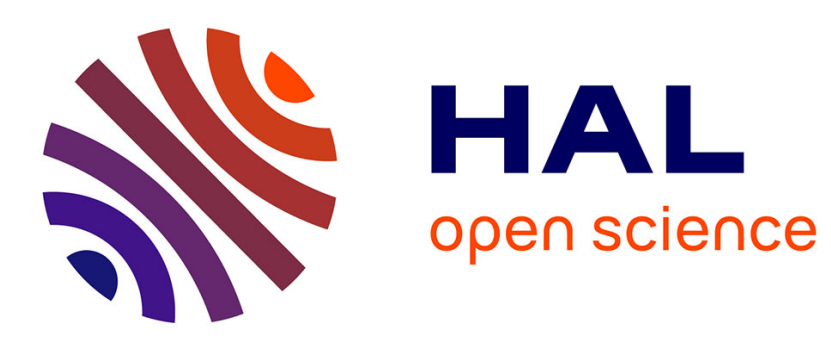

\title{
Tracking of Deformable Target in 2D Ultrasound Images
}

Lucas Royer, Maud Marchal, Anthony Le Bras, Guillaume Dardenne, Alexandre Krupa

\section{To cite this version:}

Lucas Royer, Maud Marchal, Anthony Le Bras, Guillaume Dardenne, Alexandre Krupa. Tracking of Deformable Target in 2D Ultrasound Images. Proceedings of SPIE Medical Imaging Conference, Feb 2015, Orlando, United States. hal-01080672

\section{HAL Id: hal-01080672 https://hal.science/hal-01080672}

Submitted on 5 Nov 2014

HAL is a multi-disciplinary open access archive for the deposit and dissemination of scientific research documents, whether they are published or not. The documents may come from teaching and research institutions in France or abroad, or from public or private research centers.
L'archive ouverte pluridisciplinaire HAL, est destinée au dépôt et à la diffusion de documents scientifiques de niveau recherche, publiés ou non, émanant des établissements d'enseignement et de recherche français ou étrangers, des laboratoires publics ou privés. 


\title{
Tracking of Deformable Target in 2D Ultrasound Images
}

\author{
Lucas Royer $^{1,2,3}$, Maud Marchal ${ }^{1,3}$, Anthony Le Bras ${ }^{4}$, Guillaume Dardenne ${ }^{1}$, and Alexandre Krupa ${ }^{1,2}$ \\ ${ }^{1}$ Institut de Recherche Technologique b-com, Rennes, France \\ ${ }^{2}$ Inria Rennes - Bretagne Atlantique, France \\ ${ }^{3}$ INSA de Rennes, France \\ ${ }^{4}$ CHU de Rennes, France
}

August 2, 2014

\begin{abstract}
In several medical applications such as liver or kidney biopsies, an anatomical region needs to be continuously tracked during the intervention. In this paper, we propose a novel approach for automatically tracking deformable target within $2 \mathrm{D}$ ultrasound images. Our approach uses only dense information combined with a physically-based model and has therefore the advantage of not using any fiducial marker nor a priori knowledge on the anatomical environment. The physical model is represented by a mass-spring damper system driven by different types of forces where the external forces are obtained by maximizing image similarity metric between a reference target and a deformed target across the time. This deformation is represented by a parametric warping model where the optimal parameters are estimated from the intensity variation. This warping function is well-suited to represent localized deformations in the ultrasound images because it directly links the forces applied on each mass with the motion of all the pixels in its vicinity. The internal forces constrain the deformation to physically plausible motions, and reduce the sensitivity to the speckle noise. The approach was validated on simulated and real data, both for rigid and free-form motions of soft tissues. The results are very promising since the deformable target could be tracked with a good accuracy for both types of motion. Our approach opens novel possibilities for computer-assisted interventions where deformable organs are involved and could be used as a new tool for interactive tracking of soft tissues in ultrasound images.
\end{abstract}




\section{Description of Purpose}

In several medical applications such as liver or kidney biopsies, an anatomical region needs to be continuously tracked during the intervention. Several techniques for tracking a target within ultrasound images propose to perform a registration between US images using an external localizer. For example, Xu et al. [1] acquire a freehand 3D US volume and use 2D control images tracked with a magnetic sensor mounted on the probe during the intervention. However, tracking soft tissues remains challenging due to the deformations caused by physiological motions combined with the generally weak quality of the images. Different techniques have been presented over the two last decades for non-rigid target tracking within ultrasound images. Some of them rely on a physical model or on a priori knowledge such as Dydenko et al. [2] which uses information related to the shape and the motion provided by a medical expert, or like Angelova and Mihaylova [3] where prior dynamics of a contour are considered. Shen et al. [4] proposed a registration method based on the mass-spring system in order to constrain the deformation, and Zhang et al. [5] introduced an other registration technique based on finite element model where the extraction of the scale invariant features is needed. However, their model are built from features which are difficult to extract in US images due to the speckle noise. Finally, Marami et al. [6] presented very recently an elastic registration method applicable to multi-modality image registration where the deformation is computed from modality independent neighborhood descriptor. In this paper, we propose an approach for tracking deformable target within 2D US images based on a physical model driven by smooth displacement field obtained from dense information. This allows to take into account highly localized deformation in the US images. Section 2 presents our method based on a combination of an intensity-based approach and a physically-based model. Section 3.2 describes the performances of our approach and comparisons both on simulated and on real data. Section 4 concludes the paper.

\section{Method}

The proposed approach consists in tracking a deformable target in a sequence of consecutive ultrasound images. In this section, we present our method by firstly describing how the tracking region is defined. Then, the intensity-based approach which allows to estimate the deformation is detailed. Finally, the combination between the deformable model and the intensity-based approach is explained.

\subsection{Definition of the Tracking Region}

The target is defined by a region of interest composed of $N_{p}$ pixels. A grid of $N_{c}$ control points is superimposed on it such that $N_{c}<<N_{p}$. The grid is composed of a set of blocks where each corner represents a control point. Each block includes a constant number of pixels $N_{b}$ and four controls points. A pixel position $P$ is related to the associated control points by using a bi-linear interpolation. Thus, for the vector $\mathbf{P}$ containing all the pixel positions, and the vector $\mathbf{P}_{\mathbf{c}}$ containing the control points positions, we have the following relation:

$$
\mathbf{P}=\mathbf{C} . \mathbf{P}_{\mathbf{c}}
$$

where $\mathbf{C}$ is a matrix $N_{p} \times N_{c}$ which contains all the bi-linear coefficient of each pixel position in function of its control points. The equation 1 allows to estimate the $N_{p}$ pixels positions from the control points coordinates. In this paper, our objective is to interactively find the positions of the control points such that the region of interest keeps being tracked at any time thanks to the intensity variation of each pixel.

\subsection{Intensity-based Approach}

Let us define I the vector composed of the intensity values of the $N_{p}$ pixels. The objective is to define the optimal displacement of the control points such that the error difference on the pixel intensities between the initial image and the current image is minimized. We propose to use an approach which relates the intensity time variation to the displacements of the control points by using a Jacobian $\mathbf{J}$ :

$$
\dot{\mathbf{I}}=\mathbf{J} . \dot{\mathbf{P}}_{\mathbf{c}}
$$


where $\dot{\mathbf{I}}$ is the time variation of the pixel intensities and $\dot{\mathbf{P}}_{\mathbf{c}}$ the displacements of the control points. Combining equations 1 and 2, the Jacobian can be expressed as:

$$
\mathbf{J}=\nabla \mathbf{I} \cdot \mathbf{C}
$$

where $\nabla \mathbf{I}$ represents the gradient of the pixel intensities regarding the $x$ and $y$. As it was shown in [7], if we want to ensure an exponential decrease of the error between the reference and current template, we can express an estimation of the variation of the control points $\widehat{\hat{\mathbf{P}}_{\mathbf{c}}}$ between the initial and current states as :

$$
\widehat{\mathbf{P}_{\mathbf{c}}}=-\lambda . \mathbf{J}^{+} .\left(\mathbf{I}_{\text {current }}-\mathbf{I}_{\text {ref }}\right)
$$

where $\lambda>0$ is the proportional coefficient involved in the exponential convergence decrease of the difference between the current intensities $\mathbf{I}_{\text {current }}$ and the initial ones $\mathbf{I}_{\text {ref. }} \mathbf{J}^{+}$represents the pseudo-inverse matrix of the Jacobian $\mathbf{J}$. In this work, we chose to constrain the deformation by integrating a physically-based model of the expected deformation.

\subsection{Deformable Component}

In order to take into account the deformations of the region of interest, a deformable model is superimposed to it. Our deformable model consists of a mass-spring-damper system where the nodes are the controls points of the grid. Thus, the force $F_{i j}$ exerted on a control point $P_{c}^{i}$ from a neighbor point $P_{c}^{j}$ is:

$$
F_{i j}=\left(K_{i j}\left(d_{i j}-d_{i j}^{i n i t}\right)+D_{i j}\left(\dot{P}_{c}^{i}-\dot{P}_{c}^{j}\right)\right)\left(P_{c}^{i}-P_{c}^{j}\right)
$$

where $d_{i j}$ represents the distance between the two control points in their current position. $d_{i j}^{i n i t}$ represents the initial distance value. $K_{i j}$ is the stiffness of the spring while $D_{i j}$ is the damping coefficient. The values of these coefficients can be different, depending on the homogeneities of the tissues. The forces applied on each control point $P_{c}^{i}$ of the deformable system could be summarized in $F_{i}$ :

$$
F_{i}=\sum_{j} F_{i j}+G_{i} \dot{P}_{c}^{i}+F_{e x t}
$$

where $j$ represents the index of the neighbors of the node $i$. $G_{i}$ is the velocity damping coefficient and $F_{\text {ext }}$ corresponds to the external forces. We chose to use a semi-implicit Euler integration scheme for simulating our system. The external forces are computed from the control points displacements $\widehat{\hat{\mathbf{P}}_{\mathbf{c}}}$ from equation 4 . We integrate at each time step this displacement to obtain the external force representing the intensity variation. Once the external forces have been applied to the mass-spring-damper system, the simulation is performed in order to obtain the resulting displacements of the control points.

\section{Results}

We evaluated the performances of our method both on simulated and real sequences of acquired 2D US images. The deformable targets were chosen in human liver US images acquired thanks to a 2D convex US probe of 2-5 MHz frequency bandwidth (C60, Sonosite). The tracking system is built from $\mathrm{C}++$ code, and requires a computation time of 1.4 second per frame with non-optimized code.

\subsection{Results with simulated data}

The tissue motions are reproduced by applying known rigid motions and deformations onto a fixed image. We simulated the template deformation by applying random displacements to each control point of the model described above, whereas the rigid motion is simulated by translating or rotating the whole reference image. The evaluation of our method is determined through the comparison of the transformation estimated from the undeformed template and the simulated transformation. The quality of the target tracking is assessed by measuring the distance $d$ between all the simulated and estimated control points as follows:

$$
d=\sum_{i=0}^{N_{c}} \sqrt{\left(P_{c_{i}}^{x \text { sim }}-P_{c_{i}}^{x e s t}\right)^{2}+\left(P_{c_{i}}^{y s i m}-P_{c_{i}}^{y \text { est }}\right)^{2}}
$$


where the estimated and simulated coordinates of control point $i$ are respectively $\mathbf{P}_{\mathbf{c}_{\mathbf{i}}}{ }^{\text {est }}=\left(P_{c_{i}}^{\text {est }}, P_{c_{i}}^{\text {yest }}\right)$ and $\mathbf{P}_{\mathbf{c}_{\mathbf{i}}}{ }^{\text {sim }}=\left(P_{c_{i}}^{x \text { sim }}, P_{c_{i}}^{y \text { sim }}\right)$. Our approach has been tested on two simulation scenes regarding the motion type. The results are presented on the figures 1 and 2 and show respectively the final error from the equation (7) for each image, and the evolution of the error between acquired images. We can notice from the right figure that an exponential convergence of the algorithm is obtained in 10 to 20 iterations per frame. The first scenario shown in figure 1 corresponds to the rigid motion simulation where expected convergence results are achieved. The results of a simulation that combines deformation and rigid motions are reported in the figure 2 . We can see that the distance error is slightly increased due to the normal behavior of elastic constraints which prevent the high displacement of nodes.

\subsection{Results with real data}

In order to test the robustness of both method on real data, we performed our test on a real sequence containing 173 images of $640 \times 480$ pixels. In this sequence, the target represents an hyper-echogenic area which is both deformed and displaced by natural rigid motions. Moreover, we also added an extra rigid motion which translates and rotates the original sequence. We also launched the tracking without mass-spring based only on the intensity image variation. The results are presented in figure 3 from which we can see that the presence of dynamic noise results to the mis-estimation of the deformation which makes the target tracking fails without the mass-spring system. However, we can see that the deformable target is well tracked thanks to the proposed approach.
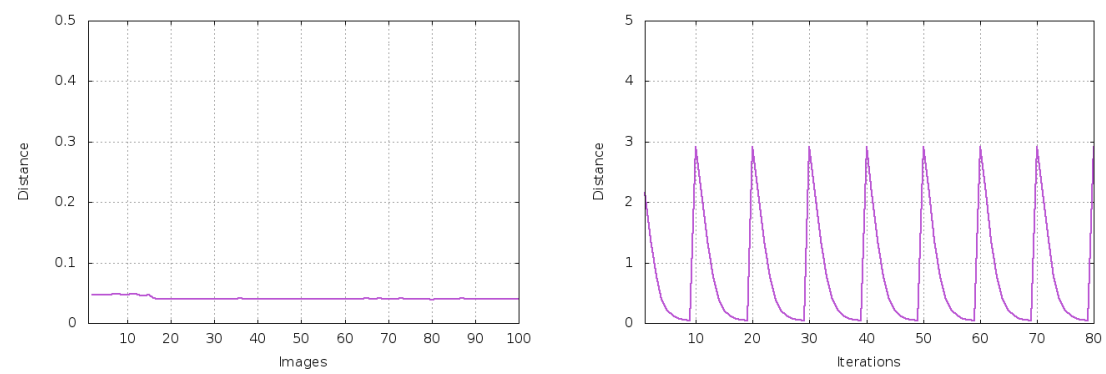

Figure 1: Rigid motion: average value of the distance $d=0.04$ pixels. (Left) Final error obtained at the convergence of the template tracking at each image acquisition, (Right) Evolution of the error between successive acquired images. The peaks are introduced by the acquisition of a new image which requires a new convergence of the grid.
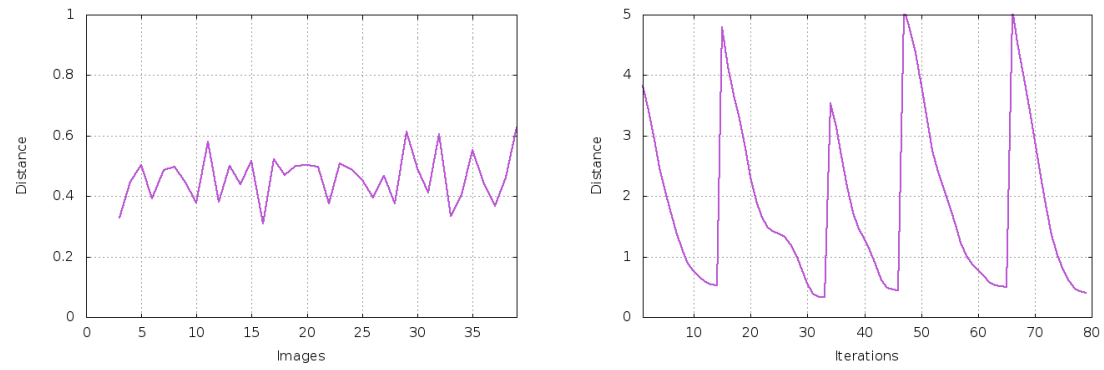

Figure 2: Rigid motion and deformation simulation: average value of the distance $d=0.47$ pixels.(Left) Final error obtained at the convergence of the template tracking at each image acquisition. (Right) Evolution of the error between successive acquired images: the peaks are introduced by the acquisition of a new image which requires a new convergence of the grid. 

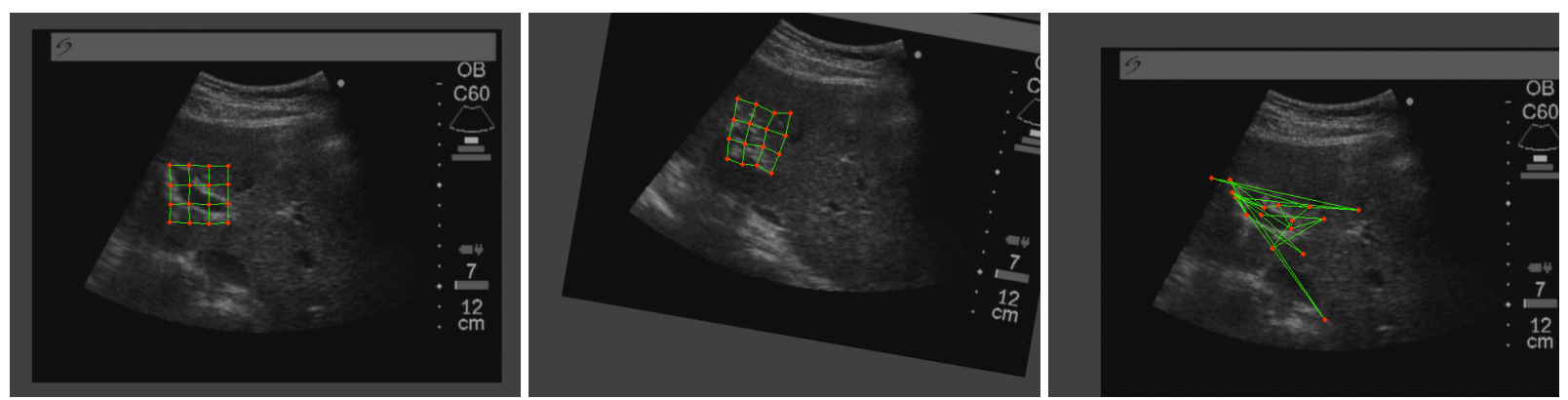

Figure 3: Results estimated on real data. (Left) Reference grid (Middle) Template tracking with mass-spring system. We can observe that the grid is deformed in order to increase the similarity between reference template and the warped template. (Right) Template tracking without mass-spring system at frame 13. For the last case, we observe tracking failure due to the speckle noise presence.

\section{Conclusion}

We proposed an interactive approach for tracking deformable target within 2D US images based only on dense information and a physically-based model. In order to estimate both the rigid and the elastic motions of the soft tissues, we introduced in our approach a physically-based model superimposed to the region of interest. This deformable model is controlled by the intensity variation of the image and allows to take into account highly localized deformations in the US images. It opens novel perspectives in computer-assisted interventions based on US imaging and where deformable organs are involved, such as image-guided needle biopsy procedures.

\section{References}

[1] S. Xu, J. Kruecker, P. Guion, N. Glossop, Z. Neeman, P. Choyke, A. K. Singh, and B. Wood, "Closed-loop control in fused mr-trus image-guided prostate biopsy,", in Proceedings of MICCAI, pp. 128-135, 2007.

[2] I. Dydenko, F. Jamal, O. Bernard, J. D’hooge, I. Magnin, and D. Friboulet, “A level set framework with a shape and motion prior for segmentation and region tracking in echocardiography," Medical image analysis, vol. 10, no. 2, pp. 162-177, 2006.

[3] D. Angelova and L. Mihaylova, "Contour segmentation in 2D ultrasound medical images with particle filtering," Machine Vision and Applications, vol. 22, no. 3, pp. 551-561, 2011.

[4] J.-K. Shen, B. J. Matuszewski, L.-K. Shark, and C. J. Moore, "Deformable image registration using spring mass system.," in BMVC (M. J. Chantler, R. B. Fisher, and E. Trucco, eds.), pp. 1199-1208, British Machine Vision Association, 2006.

[5] J. Zhang and J. Wang, "Model-based nonrigid image registration using scale-invariant features," in Proc. of IEEE International Conference on Signal Processing, Communication and Computing, pp. 1-5, 2013.

[6] B. Marami, S. Sirouspour, A. Fenster, and D. W. Capson, "Dynamic tracking of a deformable tissue based on 3d-2d mr-us image registration," in Proceedings of SPIE Medical Imaging, 2014.

[7] C. Nadeau and A. Krupa, "Intensity-based ultrasound visual servoing: Modeling and validation with 2-d and 3-d probes," IEEE Transactions on Robotics, vol. 29, no. 4, pp. 1003-1015, 2013. 